\title{
Long term ionization effect during several GLE events of solar cycle 23 - comparative analysis
}

\author{
Alexander Mishev* \\ Space Climate Research Unit, University of Oulu, Oulu, Finland. \\ E-mail: alexander.mishev@oulu.fi
}

\author{
Peter I.Y. Velinov \\ Institute for Space Research and Technology, Bulgarian Academy of Sciences, Sofia, Bulgaria. \\ E-mail: pvelinov@bas.bg
}

\begin{abstract}
It is known that the galactic cosmic rays are the main source of ionization in the Earth's stratosphere and troposphere. Occasionally, with greater probability during solar maximum periods eruptive solar processes, namely solar flares and coronal mass ejections (CMEs) lead to a production of high energy solar energetic particles (SEPs). Special class of events, called ground level enhancements (GLEs) can drastically enhance the atmospheric ionization. The induced by primary cosmic ray particles ionization is important in various processes related to atmospheric physics and chemistry, specifically the minor constituents. During the Solar cycle 23 (1996 May - 2008 January) we observed several strong GLEs, namely the Bastille day event on 14 July 2000 (GLE 59), the Easter event on 15 April 2001 (GLE 60), October-November 2003 Halloween events (GLEs 65, 66 and 67), mid-January 2005 (GLE 68 and 69) and the last event from the cycle on 13 December 2006 (GLE 70) (gle.oulu.fi). Here we study the long term ionization effect of two of the mentioned events (specifically GLE 59 and GLE 70) and perform a comparative analysis, studying the ionization in the tropospheric region. We use a full Monte Carlo 3-D model and compute the cosmic ray induced ionization as a function of the altitude above the sea level. Ionization maps of several altitudes in the troposphere and stratosphere are presented. The investigated ionization effects are much more essential at the altitudes above $12 \mathrm{~km}$ a.s.l., and especially in the Regener-Pfotzer maximum (15-20 km) and marginal at altitudes below $8 \mathrm{~km}$.
\end{abstract}

35th International Cosmic Ray Conference - ICRC 2017-

10-20 July, 2017

Bexco, Busan, Korea

${ }^{*}$ Speaker. 


\section{Introduction}

The main source of ionization in the troposphere and stratosphere of the Earth is due to high energy particles of various populations, the most important of which are the galactic cosmic rays (GCRs) [1, 2, 3, 4]. High energy GCR particles penetrate deep into the atmosphere and interacting with the air molecules induce a complicated nuclear-electromagnetic-muon cascade. This leads to an ionization of the ambient air $[1,2,4,5,6]$. The maximum of ion production in the atmosphere, observed at the altitude of about 12-15 km above the sea level (a.s.l.) is known as Regener-Pfotzer maximum $[7,8,9]$. The majority of GCRs are protons and $\alpha$-particles, and minor quantities of heavier nuclei are also observed $[10,11]$. Their flux is slightly modulated in the Heliosphere by the solar wind and follows in anti-phase the 11-year solar cycle. It also responds to transient phenomena e.g. Forbush decreases [12]. During strong eruptive solar processes as solar flares and CMEs are produced SEPs (see [13, 14, 15] and references therein). Occasionally, SEPs are accelerated to energies of about $\mathrm{GeV} /$ nucleon, which are enough to initiate an atmospheric cascade similarly to GCRs, and the secondaries eventually observed at ground level. This special class of events are known as GLEs. They significantly increase the ion production in the atmosphere, specifically in polar regions $[16,17,18,19]$. Here, we compare the ionization effect due to GLE particles in a medium to long time scales during two strong GLEs, namely GLE 59 on 14 July 2000 (Bastille day event) and GLE 70 on 13 December 2006.

\section{Model for computation of ion production rate in the atmosphere due to CRs}

The computations of ion production rate and the corresponding ionization effect are performed with a model similar to [20]. The details are given elsewhere [21, 22, 23]. The ion production rate is given by:

$$
q(h, E)=\frac{1}{E_{\text {ion }}} \sum_{i} \int_{E_{\text {cut }}}^{\infty} \int_{\Omega} \frac{\partial E(h, E)}{\partial h} D_{i}(E) \rho(h) d E d \Omega
$$

where $\partial E$ is the deposited energy in an atmospheric layer $\partial h, h$ is the air overburden (air mass) above a given altitude in the atmosphere expressed in $\mathrm{g} / \mathrm{cm}^{2}$ subsequently converted to altitude a.s.l., $D_{i}(E)$ is the differential cosmic ray spectrum for a given nuclei of primary $\mathrm{CR}, \rho$ is the

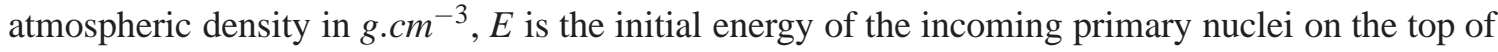
the atmosphere, $\Omega$ is the geometry factor - a solid angle and $E_{i o n}=35 \mathrm{eV}$ is the energy necessary for creation of an ion pair in air [24]. The integration is over the kinetic energy above $E_{\text {cut }}\left(R_{c}\right)$, which is defined by the local rigidity cut-off $R_{c}$ for a nuclei of type $i$ at a given geographic location by the expression $E_{c u t, i}=\sqrt{\left(\frac{Z_{i}}{A_{i}}\right)^{2} R_{c}^{2}+E_{0}^{2}}-E_{0}$, where $E_{0}=0.938 \mathrm{GeV}$ is the proton's rest mass.

The ionization effect in the stratosphere and troposphere is computed using the Eq. (2.1) averaged over the corresponding period ( $24 h$ or one week). During strong GLE events the ion production rate in the atmosphere is a superposition of the contribution of GCRs, which in some cases could be reduced (Forbush decrease) and SEPs, the latter typically with essential anisotropic part, specifically during the event onset. For the GCR spectrum we assume the force field model according to $[25,26]$, where the parametrization of local interstellar spectrum is considered as in 
[20]. The modulation potential is calculated according to [27]. For the SEPs in equation (2.1) we use a compilation of derived form ground based measurements with NMs spectra [28, 29, 30].

\section{Ion production rate during GLE 59 and GLE 70}

During the Solar cycle 23 (1996 May - 2008 January) we observed several strong GLEs, namely the Bastille day event on 14 July 2000 (GLE 59), the Easter event on 15 April 2001 (GLE 60), October-November 2003 Halloween events (GLEs 65, 66 and 67), mid-January 2005 (GLE 68 and 69) and the last event from the cycle on 13 December 2006 (GLE 70) [31, 32].
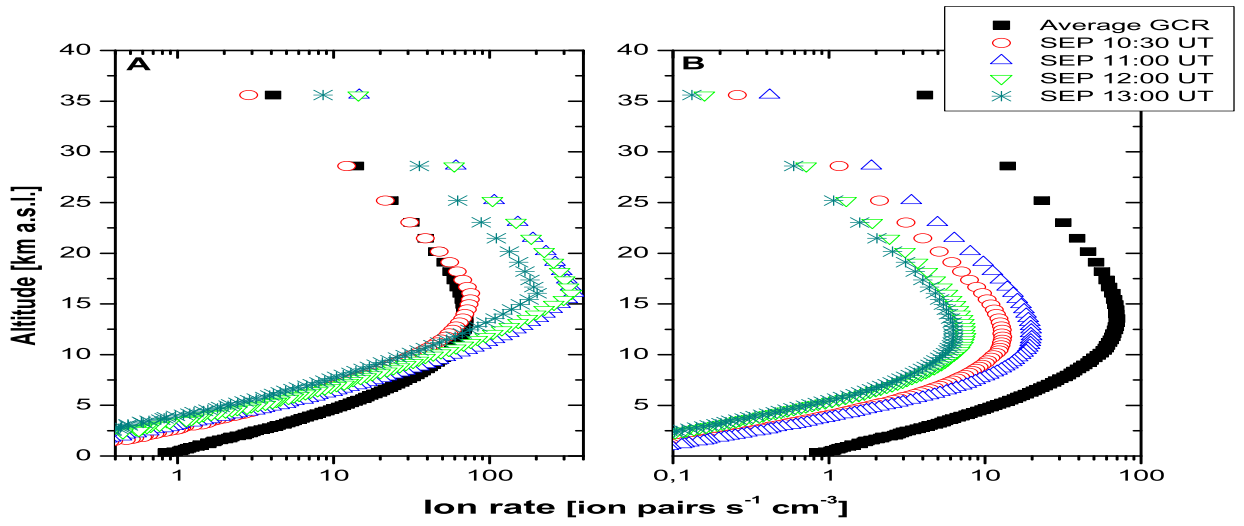

Figure 1: Ion production rate during the Bastille day GLE event on 14 July 2000. a) Ion production rate in the region with $R_{c} \leq 1 \mathrm{GV}$; b) Ion production rate in the region with $R_{c} \leq 2 \mathrm{GV}$

July 2000 was period of intense solar activity, producing three X-class flares and two halo CMEs. The GLE 59 was related to the Bastille day X5.8/3B solar flare and associated full halo CME [33]. The event onset was between 10:30 and 10:35 UT. The strongest NM increases were observed at South Pole (58.3 \%) and SANAE (54.4 \%). Here, we consider SEPs spectra and anisotropy according to reconstructions from NM data [28, 30]. The computations are fulfilled at realistic conditions, namely assuming a summer atmospheric profile [34, 35] for the Northern hemisphere and winter for the Southern hemisphere, respectively. The computed ion production rates are presented in Fig.1a for $R_{c} \leq 1 \mathrm{GV}$ cut-off, accordingly Fig.1b for $R_{c} \leq 2 \mathrm{GV}$ [36].

The mid December 2006 was characterized as period near to the minimum of solar activity. However, on $13^{\text {th }}$ of December 2006, was observed a X3.4/4 B solar flare associated with Type II and Type IV radio bursts and a fast full-halo CME. It was accompanied by a major proton event with energy producing a GLE 70 (onset at $\sim 2: 48$ UT) (maximum at Oulu and Apatity NMs $~$ $90 \%$ ). The event was characterized by a large anisotropy in its initial phase [37]. In addition, the event occurred during the recovery phase of a Forbush decrease. Therefore for ion production we consider reduced GCR flux adjusted from Oulu NM measurements. The spectral and angular characteristics of SEPs are adopted from the works of [29, 30]. Accordingly, the computed ion production rates are presented in Fig.2a for $R_{c} \leq 1 \mathrm{GV}$ cut-off and Fig.2b for $R_{c} \leq 2 \mathrm{GV}$ [38]. 


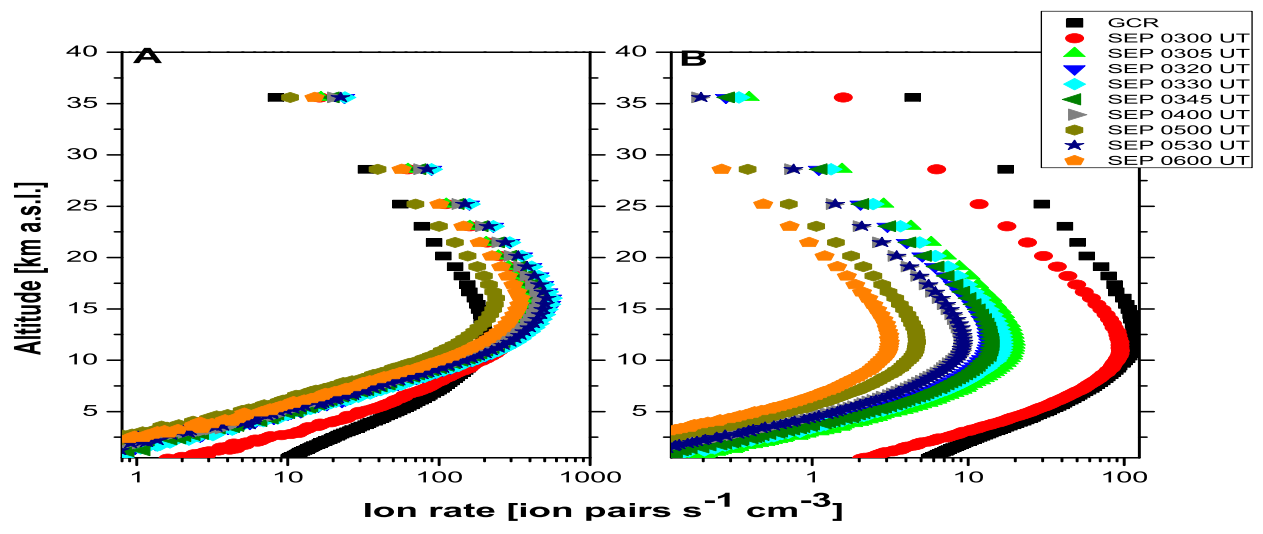

Figure 2: Ion production rate during GLE 70 on 13 December 2006. a) Ion production rate in the region with $R_{c} \leq 1 \mathrm{GV}$; b) Ion production rate in the region with $R_{c} \leq 2 \mathrm{GV}$

\section{Mid and long term term ionization effect during GLE 59 and GLE 70}

The derived ion production rates (Fig.1 and Fig.2) allow us to compute the ionization effect by integration of ion production rate over the event and/or various periods [39, 40, 41]. The $24^{h}$ ionization effect relative to the average due to GCRs at altitude of $12 \mathrm{~km}$ a.s.1., which corresponds to nearly maximal ion production level, is presented in Fig.3 in the regions with $R_{c} \leq 1 \mathrm{GV}$ rigidity cut-off, accordingly in Fig. 4 at altitude of 8 km a.s.1.
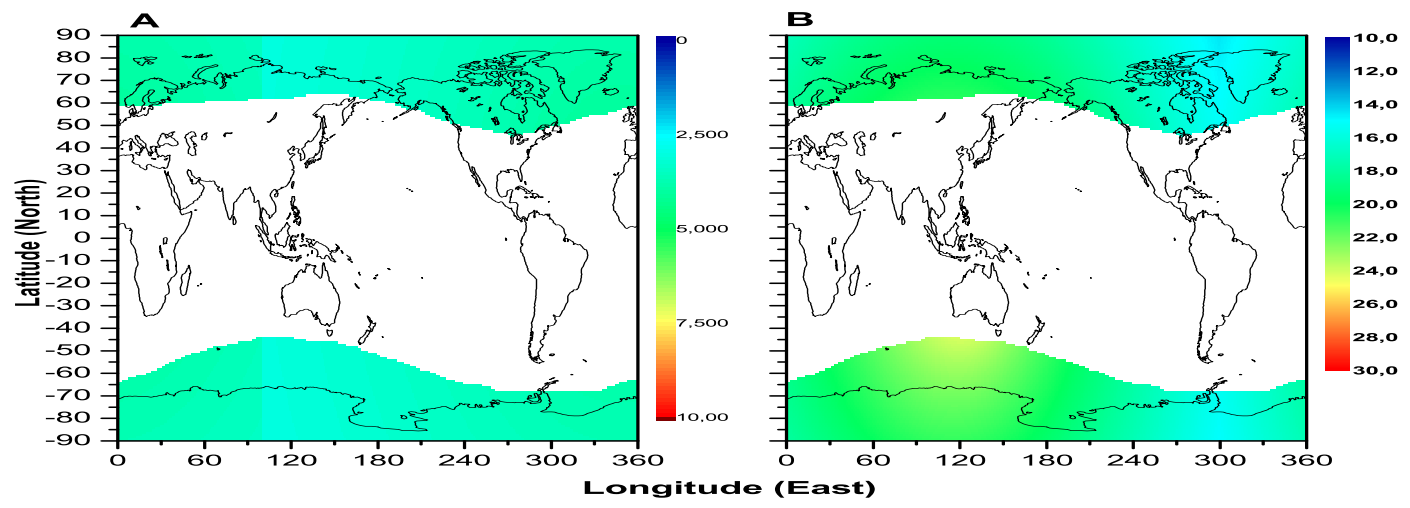

Figure 3: The $24^{h}$ ionization effect at altitude of $12 \mathrm{~km}$ a.s.l. at $R_{c} \leq 1 \mathrm{GV}$. a) The ionization effect during the Bastille day GLE on 14 July 2000; b) The ionization effect during the GLE 70 on 13 December 2006

The ion production and the corresponding ionization effect during major GLEs considerably vary throughout the event, mostly due on variation of spectral (SEP spectrum soften during the event) and angular characteristics (the pitch angle distribution broaden out). The anisotropy of SEPs as well as the duration of the event and/or the occurrence of a Forbush effect considerably affect the magnitude of ionization in a given geographic region.

Bastille day event isotropizes relatively fast, hence this effect is not as important compared to 


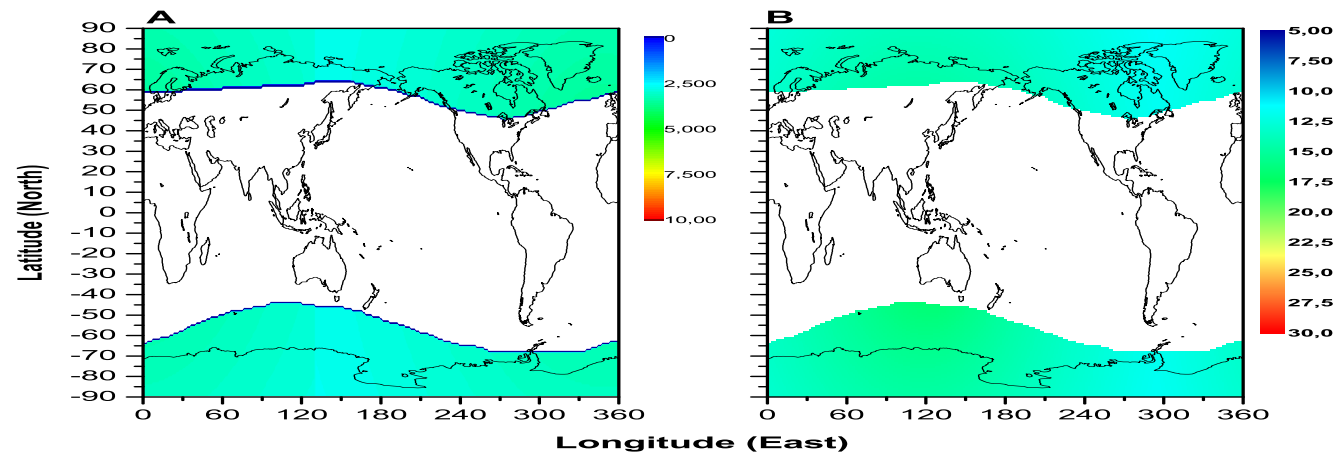

Figure 4: The $24^{h}$ ionization effect at altitude of $8 \mathrm{~km}$ a.s.l. at $R_{c} \leq 1 \mathrm{GV}$. a) The ionization effect during the Bastille day GLE on 14 July 2000; b) The ionization effect during the GLE 70 on 13 December 2006
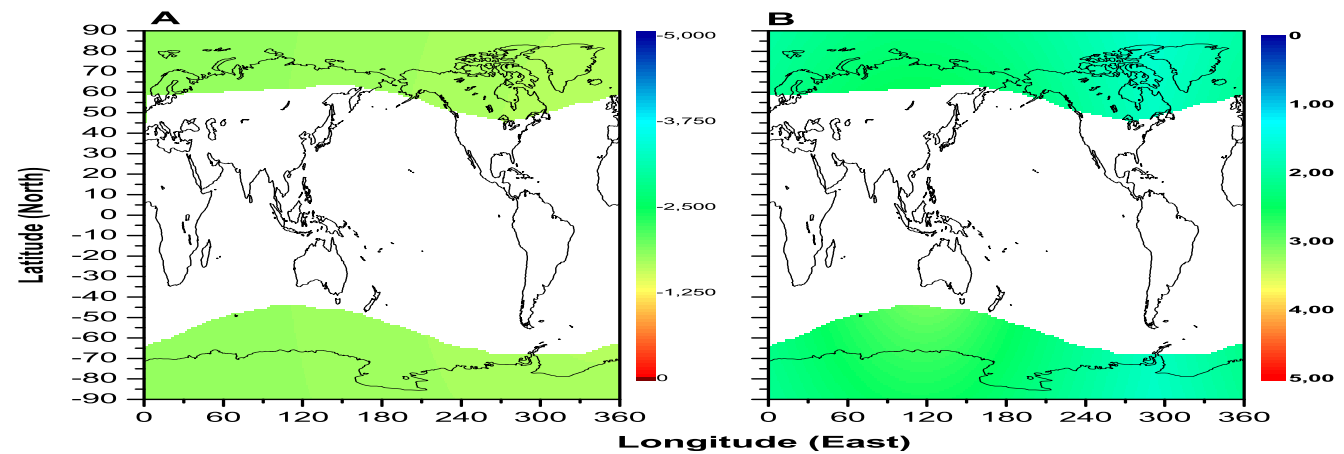

Figure 5: The weekly ionization effect at altitude of $12 \mathrm{~km}$ a.s.l. at $R_{c} \leq 1 \mathrm{GV}$. a) The ionization effect during the Bastille day GLE on 14 July 2000; b) The ionization effect during the GLE 70 on 13 December 2006

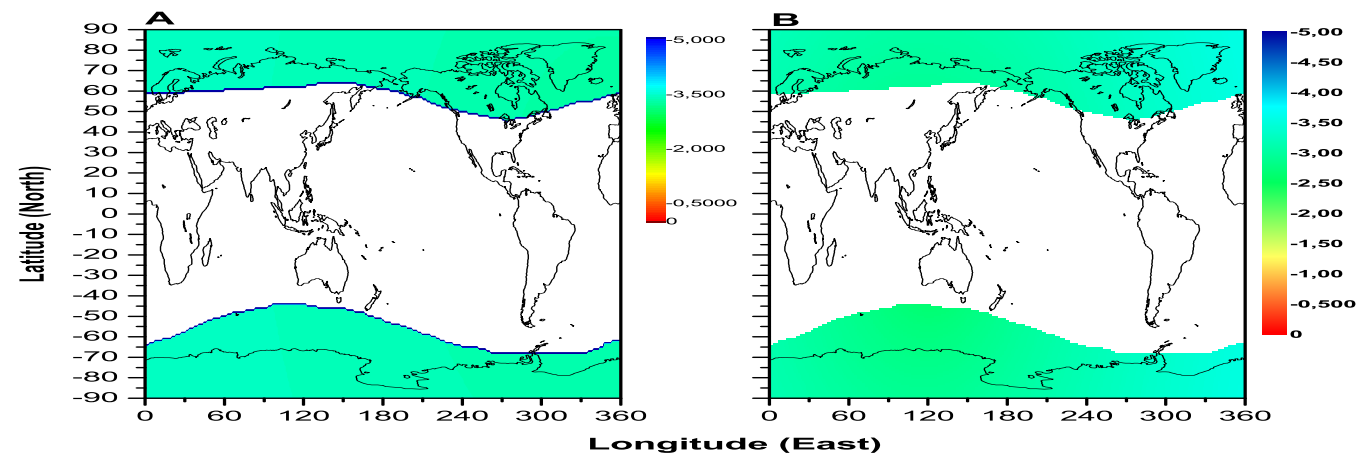

Figure 6: The weekly averaged ionization effect at altitude of $8 \mathrm{~km}$ a.s.l. at $R_{c} \leq 1 \mathrm{GV}$. a) The ionization effect during the Bastille day GLE on 14 July 2000; b) The ionization effect during the GLE 70 on 13 December 2006 
other events (Fig.3a and Fig.4a). The effect of anisotropy is clearly seen during the GLE 70 event. In general, the $24^{h}$ ionization effect at altitude of $12 \mathrm{~km}$ a.s.l. is stronger during the GLE $70(\approx 20$ $\%)$ compared to GLE $59(\approx 5 \%)$, with clearly observed anisotropic feature at Southern sub-polar region of $60-180^{\circ} \mathrm{E}$. The $24^{h}$ ionization effect at altitude of $8 \mathrm{~km}$ a.s.l. is smaller without clearly seen anisotropy. The weekly averaged ionization effect is presented in Fig. 5 (12 km a.s.l.) and Fig. 6 (8 km a.s.l.). The weekly ionization effect during the Bastille day event is slightly negative because the Forbush effect, while during the GLE 70 remains marginal, but positive. Both effects are negative in the middle troposphere at altitudes of about $8 \mathrm{~km}$ a.s.1.. In both cases there is no anisotropic features on long term time scales.

\section{Conclusion}

In this study we presented computation of medium and long term ionization effect due to CRs of galactic and solar origin during the Bastille day GLE event on 14 July 2000 and GLE 70 on 13 December 2006. It was shown that in the middle troposphere ( $8 \mathrm{~km}$ a.s.l.) the ionization effect is very similar for both events despite their difference as spectra, duration and amplitude. Moreover, the weekly effect is clearly negative, because the accompanying Forbush effect in both events. The estimated ionization effect is important for recent studies related to impact of CRs on atmospheric chemistry and physics.

\section{Acknowledgements}

This work was supported by the Center of Excellence ReSoLVE (project No. 272157) of the Academy of Finland. We acknowledge the PI of Oulu NM station.

\section{References}

[1] G. A. Bazilevskaya, I. G. Usoskin, E. Flückiger et al., Cosmic ray induced ion production in the atmosphere, Space Science Reviews 137 (2008) 149-173.

[2] I. G. Usoskin, L. Desorgher, P. Velinov et al., Ionization of the Earth's atmosphere by solar and galactic cosmic rays, Acta Geophysica 57 (2009), no. 1 88-101.

[3] P. Velinov, S. Asenovski, K. Kudela, J. Lastovička, L. Mateev, A. Mishev and P. Tonev, Impact of cosmic rays and solar energetic particles on the Earth's ionosphere and atmosphere, Journal of Space Weather and Space Climate 3 (2013) A14.

[4] I. Mironova, K. Aplin, F. Arnold, G. Bazilevskaya, R. Harrison, A. Krivolutsky, K. Nicoll, E. Rozanov, E. Turunen and I. Usoskin, Energetic particle influence on the Earth's atmosphere, Space Science Reviews (2015) 96.

[5] K. O'Brien, Calculated cosmic ray ionization in the lower atmosphere, Journal of Geophysical Research 75 (1970), no. 22 4357-4359.

[6] L. Dorman, Cosmic Rays in the Earth's Atmosphere and Underground. Kluwer Academic Publishers, Dordrecht, 2004.

[7] E. Regener and G. Pfotzer, Messungen der Ultrastrahlung in der oberen Atmosphäre mit dem Zählrohr (in german), Zeitschrift für Physik 35 (1934) 779-784. 
[8] E. Regener and G. Pfotzer, Vertical intensity of cosmic rays by threefold coincidences in the stratosphere, Nature 136 (1935), no. 3444 718-719.

[9] G. Pfotzer, Dreifachkoinzidenzen der Ultrastrahlung aus vertikaler Richtung in der Stratosphäre, Zeitschrift für Physik 102 (1936), no. 1-2 23-40.

[10] T. K. Gaisser and T. Stanev, Cosmic rays, in Review of Particle Physics (K. Nakamura et al., ), Journal of Physics G 37, (2010), 269-275.

[11] O. Adriani, G. Barbarino, G. Bazilevskaya et al., Measurements of cosmic-ray hydrogen and helium isotopes with the PAMELA experiment, Astrophysical Journal 818 (2016), no. 1.

[12] S. Forbush, On the effects in cosmic-ray intensity observed during the recent magnetic storm, Physical Review 51 (1937), no. 12 1108-1109.

[13] D. Reames, Particle acceleration at the Sun and in the Heliosphere, Space Science Reviews 90 (1999), no. 3-4 413-491.

[14] M. Aschwanden, GeV particle acceleration in solar flares and ground level enhancement (GLE) events, Space Science Reviews 171 (2012), no. 1-4 3-21.

[15] L. Miroshnichenko, Solar Cosmic Rays: Fundamentals and Applications. Springer, Dordrecht, 2015.

[16] A. Mishev, P. Velinov, L. Mateev and Y. Tassev, Ionization effect of solar protons in the Earth atmosphere - case study of the 20 January 2005 SEP event, Advances of Space Research 48 (2011), no. 7 1232-1237.

[17] I. Usoskin, G. Kovaltsov, I. Mironova, A. Tylka and W. Dietrich, Ionization effect of solar particle GLE events in low and middle atmosphere, Atmospheric Chemistry and Physics 11 (2011) 1979-1988.

[18] A. Mishev, P. Velinov, L. Mateev and Y. Tassev, Ionization effect of nuclei with solar and galactic origin in the Earth atmosphere during GLE 69 on 20 January 2005, Journal of Atmospheric and Solar-Terrestrial Physics 89 (2013), no. 1 1-7.

[19] I. Mironova, I. Usoskin, G. Kovaltsov and S. Petelina, Possible effect of extreme solar energetic particle event of 20 January 2005 on polar stratospheric aerosols: Direct observational evidence, Atmospheric Chemistry and Physics 12 (2012), no. 2 769-778.

[20] I. Usoskin and G. Kovaltsov, Cosmic ray induced ionization in the atmosphere: Full modeling and practical applications, Journal of Geophysical Research 111 (2006), no. D21206.

[21] P. Velinov, A. Mishev and L. Mateev, Model for induced ionization by galactic cosmic rays in the Earth atmosphere and ionosphere, Advances in Space Research 44 (2009), no. 9 1002-1007.

[22] A. Mishev and P. Velinov, Normalized ionization yield function for various nuclei obtained with full Monte Carlo simulations, Advances of Space Research 48 (2011), no. 1 19-24.

[23] A. Mishev and P. Velinov, Influence of hadron and atmospheric models on computation of cosmic ray ionization in the atmosphere-extension to heavy nuclei, Journal of Atmospheric and Solar-Terrestrial Physics 120 (2014) 111-120.

[24] H. Porter, C. Jackman and A. Green, Efficiencies for production of atomic nitrogen and oxygen by relativistic proton impact in air, The Journal of Chemical Physics 65 (1976), no. 1 154-167.

[25] L. Gleeson and W. Axford, Solar modulation of galactic cosmic rays, Astrophysical Journal 154 (1968) 1011-1026. 
[26] R. Burger, M. Potgieter and B. Heber, Rigidity dependence of cosmic ray proton latitudinal gradients measured by the Ulysses spacecraft: Implication for the diffusion tensor, Journal of Geophysical Research 105 (2000) 27447-27445.

[27] I. Usoskin, G. Bazilevskaya and G. Kovaltsov, Solar modulation parameter for cosmic rays since 1936 reconstructed from ground-based neutron monitors and ionization chambers, Journal of Geophysical Research 116 (2011) A02104.

[28] D. Bombardieri, M. Duldig, K. Michael and J. Humble, Relativistic proton production during the 2000 July 14 solar event: The case for multiple source mechanisms, Astrophysical Journal 644 (2006), no. 1 565-574.

[29] E. Vashenyuk, Y. Balabin, J. Perez-Peraza, A. Gallegos-Cruz and L. Miroshnichenko, Some features of the sources of relativistic particles at the sun in the solar cycles 21-23, Advances Space Research 38 (2006), no. 3 411-417.

[30] A. Mishev and I. Usoskin, Analysis of the ground level enhancements on 14 July 2000 and on 13 December 2006 using neutron monitor data, Solar Physics 291 (2016), no. 4 1225-1239.

[31] N. Gopalswamy, H. Xie, S. Yashiro, S. Akiyama, P. Mäkelä and I. Usoskin, Properties of ground level enhancement events and the associated solar eruptions during solar cycle 23, Space Science Reviews 171 (2012), no. 1-4 23-60.

[32] H. Moraal and K. McCracken, The time structure of ground level enhancements in solar cycle 23, Space Science Reviews 171 (2012), no. 1-4 85-95.

[33] K.-L. Klein, G. Trottet, P. Lantos and J.-P. Delaboudinière, Coronal electron acceleration and relativistic proton production during the 14 July 2000 flare and CME, Astronomy and Astrophysics 373 (2001), no. 3 1073-1082.

[34] A. Mishev and P. Velinov, Effects of atmospheric profile variations on yield ionization function y in the atmosphere, C. R. Acad. Bulg. Sci. 61 (2008), no. 5 639-644.

[35] A. Mishev and P. Velinov, The effect of model assumptions on computations of cosmic ray induced ionization in the atmosphere, Journal of Atmospheric and Solar-Terrestrial Physics 72 (2010), no. 5-6 476-481.

[36] A. Mishev and P. Velinov, Ionzation rate profiles due to solar and galactic cosmic rays during GLE 59 on Bastille day 14 July 2000, C. R. Acad. Bulg. Sci. 68 (2015), no. 3 359-366.

[37] R. Bütikofer, E. Flückiger, L. Desorgher, M. Moser and B. Pirard, The solar cosmic ray ground-level enhancements on 20 January 2005 and 13 December 2006, Advances in Space Research 43 (2009), no. 4 499-503.

[38] A. Mishev and P. Velinov, A maverick GLE 70 in solar minimum. calculations of enhanced ionization in the atmosphere due to relativistic solar energetic particles, C. R. Acad. Bulg. Sci. 66 (2013), no. 10 1457-1462.

[39] A. Mishev and P. Velinov, Determination of medium time scale ionization effects at various altitudes in the stratosphere and troposphere during ground level enhancement due to solar cosmic rays on 13.12.2006 (GLE 70), C. R. Acad. Bulg. Sci. 68 (2015), no. 11 1425-1430.

[40] A. Mishev and P. Velinov, Time evolution of ionization effect due to cosmic rays in terrestrial atmosphere during GLE 70, Journal of Atmospheric and Solar-Terrestrial Physics 129 (2015) 78-86.

[41] A. Mishev and P. Velinov, Ionization effect due to cosmic rays during bastille day event - GLE 59 on short and mid time scales, C. R. Acad. Bulg. Sci. 69 (2016), no. 11 1479-1484. 